\title{
Dynamic Mechanical Properties of Vietnam Modified Natural Rubber via Grafting with Styrene
}

\author{
T. A. Dung, ${ }^{1}$ N. T. Nhan, ${ }^{1}$ N. T. Thuong, ${ }^{1}$ D. Q. Viet, ${ }^{1}$ N. H. Tung, ${ }^{1}$ \\ P. T. Nghia, ${ }^{1}$ S. Kawahara, ${ }^{2}$ and T. T. Thuy ${ }^{1}$ \\ ${ }^{1}$ School of Chemical Engineering, Hanoi University of Science and Technology, No. 1, Dai Co Viet Street, Hai Ba Trung District, \\ Hanoi, Vietnam \\ ${ }^{2}$ Department of Materials Science and Technology, Faculty of Engineering, Nagaoka University of Technology, \\ 1603-1 Kamitomioka-machi, Nagaoka, Niigata, Niigata 940-2188, Japan
}

Correspondence should be addressed to T. T. Thuy; thuy.tranthi3@hust.edu.vn

Received 16 October 2017; Accepted 10 December 2017; Published 27 December 2017

Academic Editor: Bernabé L. Rivas

Copyright (C) 2017 T. A. Dung et al. This is an open access article distributed under the Creative Commons Attribution License, which permits unrestricted use, distribution, and reproduction in any medium, provided the original work is properly cited.

\begin{abstract}
The dynamic mechanical behavior of modified deproteinized natural rubber (DPNR) prepared by graft copolymerization with various styrene contents was investigated at a wide range of temperatures. Graft copolymerization of styrene onto DPNR was performed in latex stage using tert-butyl hydroperoxide (TBHPO) and tetraethylene pentamine (TEPA) as redox initiator. The mechanical properties were measured by tensile test and the viscoelastic properties of the resulting graft copolymers at wide range of temperature and frequency were investigated. It was found that the tensile strength depends on the grafted polystyrene; meanwhile the dynamic mechanical properties of the modification of DPNR meaningfully improved with the increasing of both homopolystyrene and grafted polystyrene compared to DPNR. The dynamic mechanical properties of graft copolymer over a large time scale were studied by constructing the master curves. The value of $b_{T}$ has been used to prove the energetic and entropic elasticity of the graft copolymer.
\end{abstract}

\section{Introduction}

With the progressive increase of global product demands and the continuous decrease of worldwide fossil resources alongside with the growing concerns about environmental pollutions and carbon emissions, it is very crucial to reduce the dependence on the nonrenewable fossil resources [1-3]. Natural rubber (NR) is one of the most valuable renewable resources. It has attracted great interest in many fields, such as in transportation and industrial and medical sectors, mainly due to outstanding properties such as high tensile strength, high elongation, and high elasticity [4-9].

Although NR possesses many superior properties, it lacks some properties such as oil, weather, and abrasion resistances. Consequently, it has often been modified by chemical the techniques such as graft copolymerization or the physical techniques meet the demand requirement for other applications such as in tire industry, to prepare composites with desirable properties [10-17]. Among them, graft copolymerization is a well-established technique to prepare hybrid polymer with unique structure necessary for various applications by using different monomers. Styrene is one of the common monomer which was grafted onto natural rubber using graft copolymerization technique in latex stage or solution stage $[18,19]$. The graft copolymer of styrene onto natural rubber possesses outstanding properties compared to that of pure natural rubber. However, for the application in industrial tire, it is necessary to understand the complex mechanical behavior of the graft copolymers.

Dynamic mechanical analysis (DMA) is an important method to indicate relaxation dynamic of polymeric materials. A sinusoidal stress is applied and the strain in the material is measured, allowing one to determine the moduli. By studying the dependence of storage and loss moduli on temperature or frequency, it is possible to show the dynamic mechanical properties of the materials and their applications [20-23]. Graft copolymerization of styrene onto deproteinized natural rubber resulted in a formation of 
core-shell structure, in which natural rubber particle as a core was surrounded by polystyrene nanolayer as a shell [24]. This structure and polystyrene chain play an important role in outstanding mechanical properties of graft copolymer; for instance, the tensile strength of graft copolymer increases about 4 times that of natural rubber. The dynamic mechanical properties of graft copolymer had also been measured and the results showed that the storage modulus of graft copolymer increases about 25 times compared with that of natural rubber [24]. However, DMA measurement had been carried out in narrow range of frequencies from 0.1 to $100 \mathrm{~Hz}$ due to the limitation of the equipment. The DMA result cannot cover all dynamic behavior of graft copolymer [20-23]. To understand the effect of polystyrene chain and the structure on dynamic mechanical properties, it is obligatory to study the dynamic mechanical properties of graft copolymer over a wide frequency range or a large time scale [20-23]. Therefore, the time-temperature superposition principle has been applied to obtain master curves of the modulus for the polymer [21, 25-27].

In the present study, the dependence of dynamic mechanical properties on styrene content of the graft-copolymer has been investigated. In addition, the master curves have been built to explore the dynamic mechanical properties in over a wide frequency range or a large time scale. The horizontal and vertical shift factors, which are the important factors in the time-temperature superposition principle, are calculated to determine elasticity of graft copolymer.

\section{Experimental}

2.1. Chemicals Reagents. NR latex used was commercial high ammonia natural rubber (HANR), which was purchased from Duy Hang Company, Vietnam. Dry rubber content (DRC) of HANR was about $60 \mathrm{wt}$.\%. Sodium dodecyl sulfate (SDS, 98\%) was obtained from Chameleon, Japan. Styrene was purchased from Sigma-Aldrich. Tert-butyl hydroperoxide (68\%) and tetraethylene pentamine (TEPA, 95\%) were purchased from TCI, Japan. 2-Butanone (99\%) and acetone (99.5\%) were obtained from Merck.

2.2. Preparation of DPNR Latex and Graft Copolymer Samples. Deproteinized natural rubber (DPNR) was prepared by incubation of the latex with $0.1 \mathrm{wt} . \%$ urea and $1 \mathrm{wt} . \%$ SDS at room temperature for $60 \mathrm{~min}$ followed by centrifugation at $10000 \mathrm{rpm}$ for $30 \mathrm{~min}$. The cream fraction was redispersed in 1 wt.\% SDS solution, and it was washed twice by centrifugation to prepare the DPNR latex with $60 \mathrm{wt} . \%$ DRC. The resulting DPNR latex contained about $0.1 \mathrm{wt}$ \% SDS [28].

The graft copolymer samples are prepared by graft copolymerization using $3.5 \times 10^{-5} \mathrm{~mol}$ initiator/g dried rubber. The effect of styrene concentrations in the values of 1.0, 1.5 , and $2.0 \times 10^{-3} \mathrm{~mol} / \mathrm{g}$ dried rubber was investigated. The detail procedure for the graft copolymerization was carried out according to the following steps. The DPNR latex was first introduced into a $500 \mathrm{~cm}^{3}$ glass reactor equipped with a mechanical stirrer. The latex was first purged nitrogen for $60 \mathrm{~min}$ to remove the dissolved oxygen and stirred at a constant speed $(400 \mathrm{rpm})$. Afterwards TBHPO/TEPA were added as an initiator followed by styrene as a monomer. The reaction was carried out by stirring the latex at above $400 \mathrm{rpm}$ for 2.5 hours at room temperature $[24,29,30]$.

The unreacted styrene was removed by using a rotary evaporator under reduced pressure. The product was put in a glass petri dish and dried in a vacuum oven at $50^{\circ} \mathrm{C}$ for more than a week. Soxhlet extraction was performed to purify the product. The free polystyrene was extracted with acetone/2butanone $3: 1$ mixture under nitrogen atmosphere in the dark for $24 \mathrm{~h}$, followed by drying under reduced pressure for a week.

The styrene conversion and grafting efficiency of graft copolymerization were determined by gravimetric method [24].

2.3. Characterization. The dynamic mechanical properties of the graft copolymer were measured with an Anton Paar Physica MCR 302. Specimen was a $12 \mathrm{~mm}$ diameter parallel plate geometry. The sample thickness was $1 \mathrm{~mm}$ in order to further the shear rate in the sample. Shear deformation was measured in the temperature range from -70 to $140^{\circ} \mathrm{C}$ with the impacted frequency from 0.1 to $10 \mathrm{~s}^{-1}$.

The master curves were built following the timetemperature superposition principle. The horizontal shift factor, $a_{T}$, and vertical shift factor, $b_{T}$, are expressed by the WLF equations (1) and (2), respectively [21, 27, 31-33].

$$
\begin{aligned}
\log a_{T} & =\frac{-C_{1}\left(T-T_{r}\right)}{C_{2}+T-T_{r}}, \\
G\left(t, T_{r}\right) & =\frac{G\left(f a_{T}, T\right)}{b_{T}}
\end{aligned}
$$

where $C_{1}$ and $C_{2}$ are positive constants that depend on the material and the temperature.

Tensile measurement was conducted using a Toyo Seiki Strograph VG10E according to JIS K6251. Film samples were cut with a Dumbbell-shaped No. 7. The crosshead speed was $200 \mathrm{~mm} \mathrm{~min}^{-1}$.

\section{Results and Discussion}

3.1. Effect of Styrene Content on the Dynamic Mechanical Property of Graft Copolymers. In our previous work, it has been shown that the highest styrene conversion and grafting efficiency were achieved at $3.5 \times 10^{-5} \mathrm{~mol}$ initiator/g dried rubber and $1.5 \times 10^{-3} \mathrm{~mol}$ styrene/g dried rubber [24]. In the present study, we performed the graft copolymerization with various styrene concentrations to obtain graft copolymers that are DPNR-graft-PS, with different styrene contents. The parameters for the graft copolymerization were shown in Table 1 . The conversion and grafting efficiency were calculated by gravimetric method as mentioned in our previous work [24].

Figure 1 shows the temperature dependence of $\tan \delta$ (loss tangent) for DPNR and DPNR-graft-PS samples at $10 \mathrm{~Hz}$. The peaks at $-55^{\circ} \mathrm{C}$ and $105^{\circ} \mathrm{C}$ were characterized for glass transition temperature $\left(T_{g}\right)$ of NR and PS, respectively [18]. From the DPNR to DPNR-graft-PS 2 sample, the height of the 
TABLE 1: The conversion and grafting efficiency of the graft copolymerization.

\begin{tabular}{lccccc}
\hline Sample & $\begin{array}{c}\text { Styrene concentration } \\
\left(10^{-3} \mathrm{~mol} / \mathrm{g}\right)\end{array}$ & $\begin{array}{c}\text { Conversion } \\
(\%)\end{array}$ & $\begin{array}{c}\text { Grafting efficiency } \\
(\%)\end{array}$ & $\begin{array}{c}\text { Total styrene content } \\
(\mathrm{phr})\end{array}$ & $\begin{array}{c}\text { Grafted styrene } \\
\text { content } \\
(\mathrm{phr})\end{array}$ \\
\hline DPNR & 0 & 0 & 0 & 0 & 0 \\
DPNR-graft-PS 1 & 1.0 & 69.20 & 52.67 & 7.21 & 3.80 \\
DPNR-graft-PS 1.5 & 1.5 & 91.03 & 75.02 & 14.22 & 10.67 \\
DPNR-graft-PS 2 & 2.0 & 82.08 & 53.21 & 17.10 & 9.10 \\
\hline
\end{tabular}

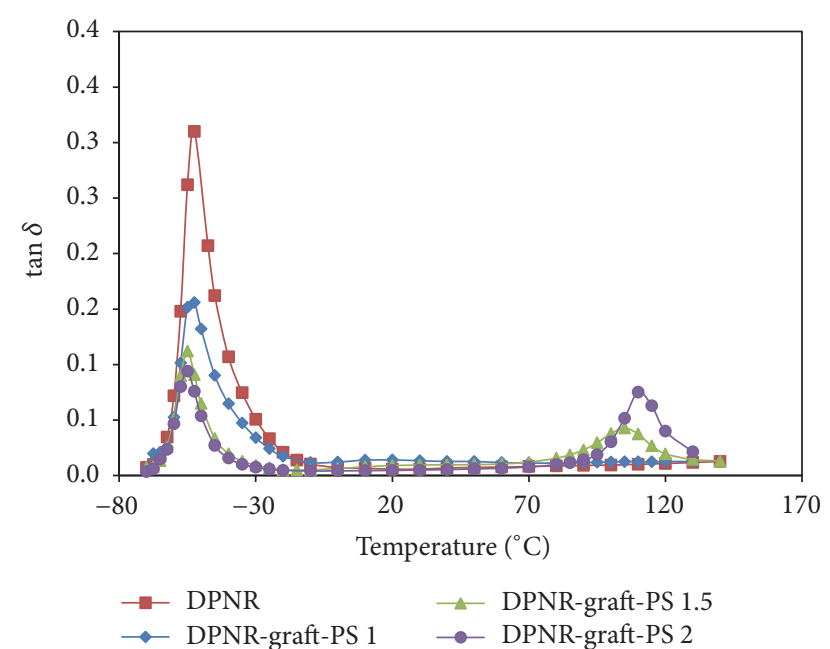

FIgURE 1: Temperature dependence of $\tan \delta$ for DPNR and DPNRgraft-PS samples at $10 \mathrm{~Hz}$.

loss tangent peak at $-55^{\circ} \mathrm{C}$ decreases, while that value at $105^{\circ} \mathrm{C}$ increases. This may be explained to be due to the increase of styrene content in the graft copolymer.

Figure 2 shows the temperature dependence of loss modulus for DPNR and DPNR-graft-PS samples. The loss modulus expresses the viscous component and the dissipation capacity of the materials. In the temperature dependence of loss modulus curves of any NR or its graft copolymerization, there are three distinct regions. At low temperature, the graft copolymer in the glassy state shows a high value of the modulus. In the transition region from the glassy state to the rubbery state, $G^{\prime \prime}$ significantly decreases to a constant value. This region is the onset of chain mobility and corresponds to the glass transition temperature $T_{g}$. At temperature higher than $T_{g}$, the value of $G^{\prime \prime}$ reaches the minimum, which is similar to that in the rubbery state. For the graft copolymer samples, an additional region appears at the temperature above $T_{g}$ of polystyrene. This region is in rubbery flow state, which is characterized by having plastic component in the material. Thus, it is obvious that the width of this region increases with the increase of styrene content.

In our previous work, the graft copolymerization resulted in the formation of core-shell structure, in which NR particles as the core were surrounded by nanolayer of polystyrene [24]. The increase of styrene content in graft copolymer led to the increase of the polystyrene layer thickness $[18,24]$. Thus, the

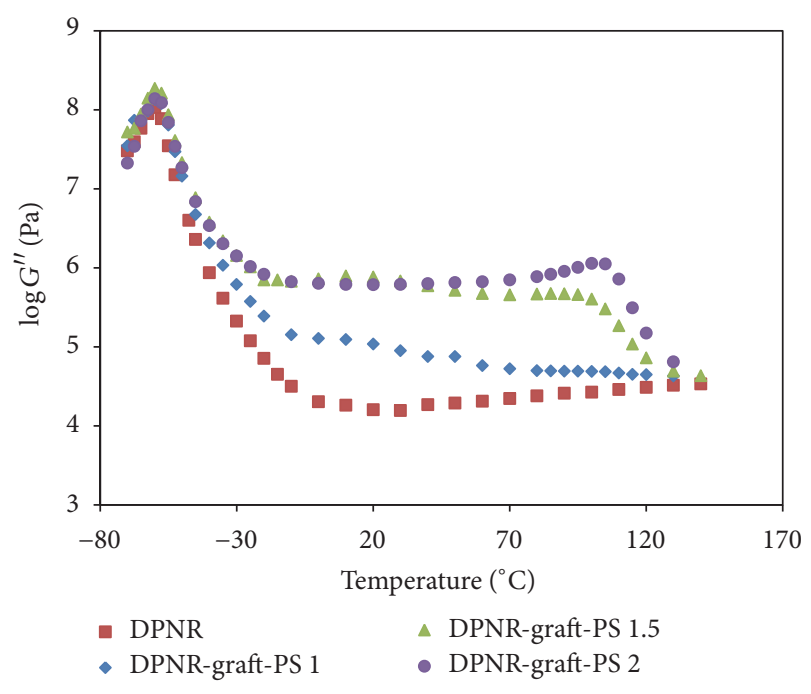

FIGURE 2: Temperature dependence of loss modulus for DPNR and DPNR-graft-PS at $10 \mathrm{~Hz}$.

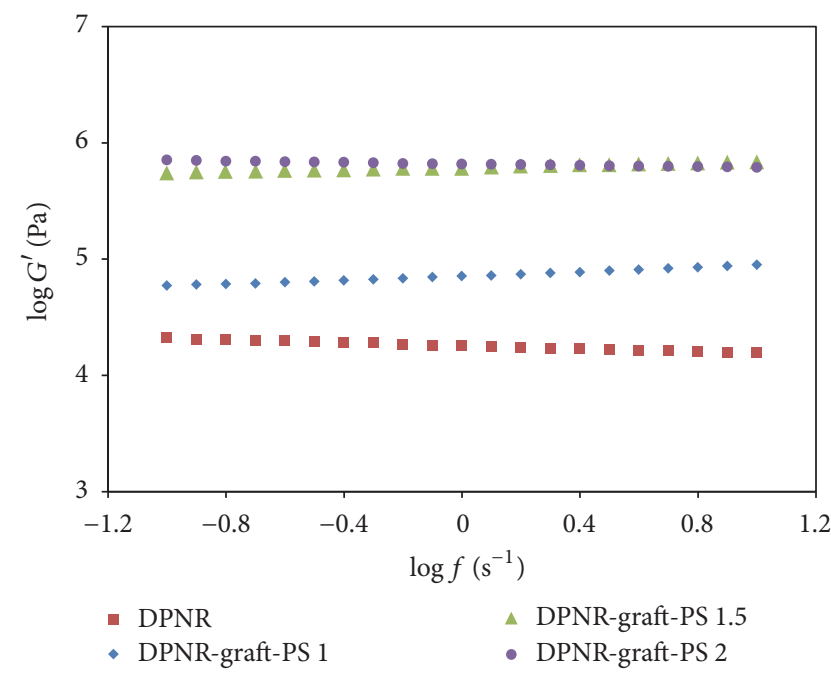

FIGURE 3: Frequency dependence of storage modulus for DPNR and DPNR-graft-PS at $30^{\circ} \mathrm{C}$.

changing in property of the graft-copolymer is explained to be due to the changing in styrene content.

Variation of the storage modulus as a function of frequency for DPNR and DPNR-graft-PS at $30^{\circ} \mathrm{C}$ is shown in Figure 3 . The storage modulus is related to the elastic modulus 


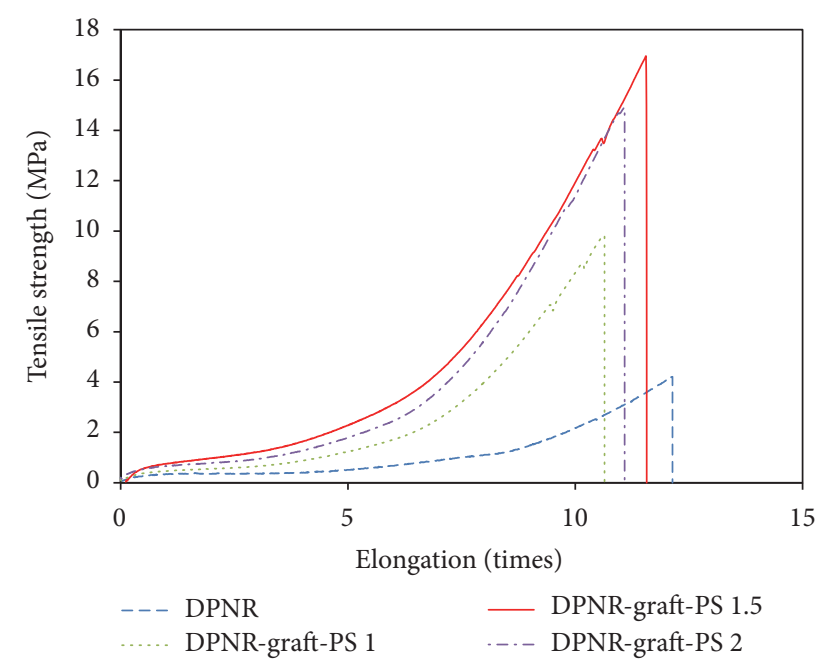

FIGURE 4: Stress-strain curves of un-vulcanized DPNR and various DPNR-graft-PS.

of polymer at the loading, in which the increase in the styrene content also resulted in the increase in storage modulus of graft copolymer. However, there is just a slight difference between storage modulus of DPNR-graft-PS 1.5 and DPNRgraft-PS 2. This may be explained to be due to the difference in amount of grafted styrene and homopolystyrene, in which the amount of grafted styrene of DPNR-graft-PS 1.5 was higher than that of DPNR-graft-PS 2, and the amount of homopolystyrene of DPNR-graft-PS 2 was higher than that of DPNR-graft-PS 1.5. The formation of homopolystyrene may have a negative effect on the property. The increase of homopolystyrene content in graft copolymer may result in the increasingly propagation of voids in the material. Because the polystyrene is harder than NR, during applying a load, the voids may be formed surrounding the polystyrene particles. When the voids are growing, it will lead to the propagation of voids and the material is collapsed. Therefore, the tensile strength of DPNR-graft-PS 1.5 was higher than that of DPNRgraft-PS 2, which is shown in Figure 4. These results suggested that not only the grafted styrene but also homopolystyrene is important factors effect on the tensile strength as well as dynamic mechanical properties of graft copolymers.

From the reasons above, the DPNR-graft-PS 1.5, which contains the highest grafted styrene content, has been selected to construct the master curves to investigate the energetic and entropic elasticity of the graft copolymers.

\subsection{Energetic and Entropic Elasticity of Graft Copolymer.} Figure 5 shows storage modulus, loss modulus, and $\tan \delta$ at various temperatures of DPNR and DPNR-graft-PS. The measurement covered from the glassy state to rubbery state. In the previous work [24], the value of the $G^{\prime}$ at the plateau region of DPNR-graft-PS was about 25 times higher than that of DPNR. Furthermore, the value $G^{\prime \prime}$ of the graft copolymer was found to be nearly independent of the frequency. It was nearly constant as the frequency increased. These led to the decreases of the value of $\tan \delta$ for DPNR-graft-PS at the low-frequency region and the increase to almost constant value at the high-frequency region. These results may be explained by the effect of polystyrene layer in the graft copolymer with the core-shell structure. Therefore, in order to elucidate the relationship between viscoelastic properties and the core-shell structure, the dependence of the viscoelastic properties on temperature and frequency was investigated by constructing the master curves.

The master curves of rubber samples were constructed based on the time-temperature superposition procedure. The line at $-65^{\circ} \mathrm{C}\left(T_{r}=-65^{\circ} \mathrm{C}\right)$ is chosen as the baseline to move the lines at different temperatures to build the master curve. The $\tan \delta$ line is used to determine the horizontal shift factor, $a_{T}$, so it is independent with the vertical shift factor, $b_{T}$. Selecting the horizontal shift factor, $a_{T}$, in temperatures to shift the $\tan \delta$ lines to the temperature $T_{r}$, we obtained the $\tan \delta$ master curves, which is shown in Figure 6.

In Figure 6, the master curves are superimposed smoothly confirming that the measurements and the superposition are reliable. On the $\tan \delta$ master curve of DPNR, there is only one peak at the large oscillatory frequency due to the NR, whereas there are two peaks on the $\tan \delta$ master curve of DPNR-graft-PS, the peak in the large oscillatory frequency attributed to the NR and the remaining peak in the small oscillation frequency described to the polystyrene chains, which is grafted onto the NR via graft copolymerization.

The $a_{T}$ value is recalculated using the WLF equation (the solid line). Then, the dependence of $a_{T}$ on temperature for DPNR and DPNR-graft-PS is shown in Figure 7. The result indicates that the $a_{T}$ for DPNR depends on temperature which is in agreement with the WLF equation. It has been proved by the value of positive constants, $C_{1}$ and $C_{2}$. These have been found to be 14.33 and 47.24 , respectively.

In the case of the island-matrix structure of rubber-filler system, the temperature dependence of $a_{T}$ is identical to mechanical relaxation for the polymer matrix. $a_{T}$ is a ratio of any relaxation time at one temperature to its value of the chosen reference temperature [27, 34-39]. In previous work, Isono et al. have reported that the horizontal shift factor $a_{T}$ of rubber filled with carbon black particles was not changed by the changing of carbon black particles amount $[36,38]$. Kawahara et al. have also reported that $a_{T}$ of natural rubber grafted with organosilane monomer was independent on the amount of silica $[39,40]$. Therefore, the recovery of the rubber-filler system is only affected by rubber. Conversely, the dependence of $a_{T}$ on temperature of DPNR-graft-PS is just in agreement with WLF equation in the temperature range from $T_{r}$ to $T_{r}+60^{\circ} \mathrm{C}$. The WLF equation cannot be extended to the higher temperature other than $T_{r}+60^{\circ} \mathrm{C}$. The different dependence of $a_{T}$ on temperature for filler rubber and graft copolymer is assumed to be due to the formation of chemical linkages between polystyrene and natural rubber that is formed via graft copolymerization. Thus, the recovery of the graft copolymer was contributed to not only NR but also polystyrene chains covering NR particles.

After obtaining the values of $a_{T}$ in the temperatures, the vertical shift factor is calculated to shift the lines $G^{\prime}$ and $G^{\prime \prime}$. The vertical shift factor is determined by formula (2). The master curves of $G^{\prime}$ and $G^{\prime \prime}$ are shown in Figures 8 and 9. 

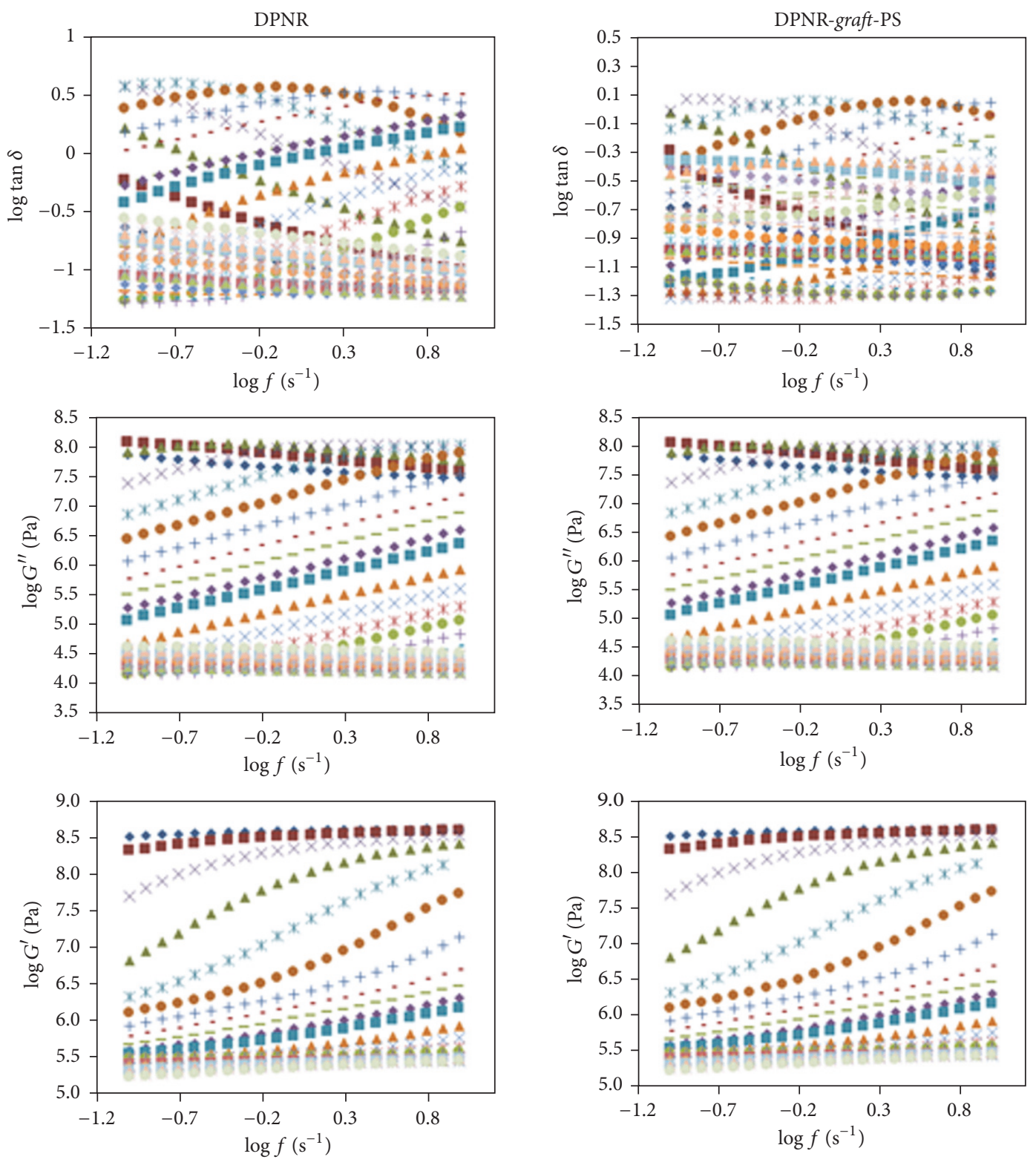

$$
\begin{array}{lll}
*-70^{\circ} \mathrm{C} & *-60^{\circ} \mathrm{C} & =-50^{\circ} \mathrm{C} \\
-40^{\circ} \mathrm{C} & +-20^{\circ} \mathrm{C} & =10^{\circ} \mathrm{C} \\
\times 40^{\circ} \mathrm{C} & -80^{\circ} \mathrm{C} & 120^{\circ} \mathrm{C} \\
=-67.5^{\circ} \mathrm{C} & --57.5^{\circ} \mathrm{C} & -47.5^{\circ} \mathrm{C} \\
\times-35^{\circ} \mathrm{C} & --15^{\circ} \mathrm{C} & -20^{\circ} \mathrm{C} \\
-50^{\circ} \mathrm{C} & * 90^{\circ} \mathrm{C} & 130^{\circ} \mathrm{C} \\
\times-65^{\circ} \mathrm{C} & +-55^{\circ} \mathrm{C} & =-45^{\circ} \mathrm{C} \\
\times 30^{\circ} \mathrm{C} & -10^{\circ} \mathrm{C} & \times 30^{\circ} \mathrm{C} \\
+60^{\circ} \mathrm{C} & =100^{\circ} \mathrm{C} & 140^{\circ} \mathrm{C} \\
--62.5^{\circ} \mathrm{C} & --52.5^{\circ} \mathrm{C} & \\
-25^{\circ} \mathrm{C} & -0{ }^{\circ} \mathrm{C} & \\
-70^{\circ} \mathrm{C} & -110^{\circ} \mathrm{C} &
\end{array}
$$

\begin{tabular}{|c|c|c|}
\hline - $-70^{\circ} \mathrm{C}$ & X. $-60^{\circ} \mathrm{C}$ & $=-50^{\circ} \mathrm{C}$ \\
\hline A $-40^{\circ} \mathrm{C}$ & $+-20^{\circ} \mathrm{C}$ & $=10^{\circ} \mathrm{C}$ \\
\hline$\times 40^{\circ} \mathrm{C}$ & $-80^{\circ} \mathrm{C}$ & $120^{\circ} \mathrm{C}$ \\
\hline$=-67.5^{\circ} \mathrm{C}$ & $-57.5^{\circ} \mathrm{C}$ & $-47.5^{\circ} \mathrm{C}$ \\
\hline$\times-35^{\circ} \mathrm{C}$ & $-15^{\circ} \mathrm{C}$ & A $20^{\circ} \mathrm{C}$ \\
\hline - $50^{\circ} \mathrm{C}$ & * $90^{\circ} \mathrm{C}$ & $\times \quad 130^{\circ} \mathrm{C}$ \\
\hline$\times-65^{\circ} \mathrm{C}$ & $+-55^{\circ} \mathrm{C}$ & $=-45^{\circ} \mathrm{C}$ \\
\hline$\times 30^{\circ} \mathrm{C}$ & $=10^{\circ} \mathrm{C}$ & $\times 30^{\circ} \mathrm{C}$ \\
\hline$+60^{\circ} \mathrm{C}$ & $=100^{\circ} \mathrm{C}$ & $=140^{\circ} \mathrm{C}$ \\
\hline$-62.5^{\circ} \mathrm{C}$ & $=-52.5^{\circ} \mathrm{C}$ & \\
\hline$-25^{\circ} \mathrm{C}$ & $+0^{\circ} \mathrm{C}$ & \\
\hline$-70^{\circ} \mathrm{C}$ & A $110^{\circ} \mathrm{C}$ & \\
\hline
\end{tabular}

FIGURE 5: Frequency sweep curves at various temperatures of DPNR and DPNR-graft-PS. 


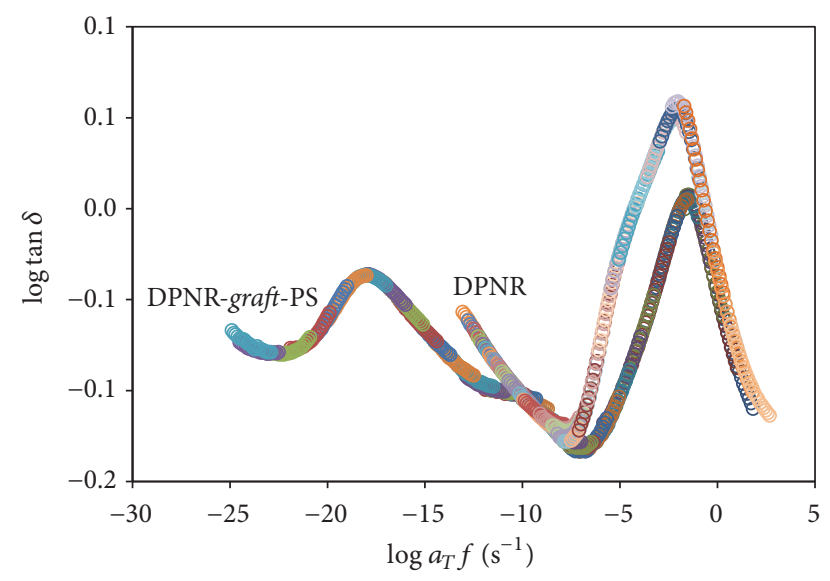

FIGURE 6: Master curves of $\tan \delta$ of DPNR and DPNR-graft-PS.

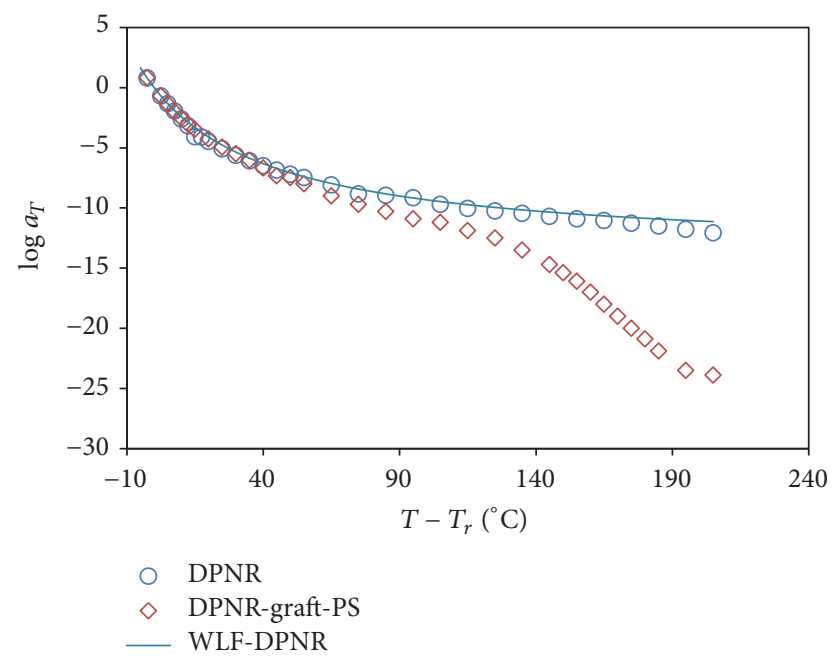

FIgURE 7: Temperature dependence of horizontal shift factor of DPNR and DPNR-graft-PS.

The master curve of $G^{\prime}$ and $G^{\prime \prime}$ showed the remarkable state transitional regions of DPNR and DPNR-graft-PS. It is obviously seen that the elasticity region of the DPNRgraft-PS samples occurred earlier and maintained longer than that of DPNR, demonstrating that the graft copolymer has longer working time than DPNR. Apart from the fact that the glassy state and elastic state of DPNR-graft-PS are the same compared to DPNR, the DPNR-graft-PS sample also has a typical state transition of the polystyrene chain.

The value of the vertical shift factor, $b_{T}$, relates to the elasticity properties of materials. Elasticity is generally divided in two parts: energetic and entropic components [21, 34-38]. The entropic component relates to an ability to return back to initial form after applying a force. The force applying on the rubber will stretch out and orient the rubber chains. This will decrease the entropy of the chains and then create an entropic force. This entropic force plays a role in driving the rubber to initial form. Besides that, the energetic force associates with the intra- and intermolecular energy. In natural rubber, the intermolecular energy is approximate to zero, so the natural

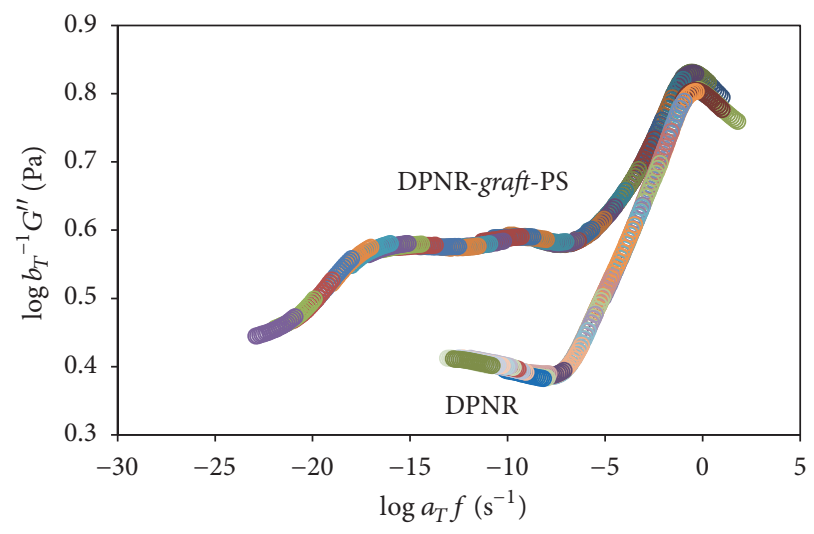

FIGURE 8: Master curves of loss modulus for DPNR and DPNRgraft-PS.

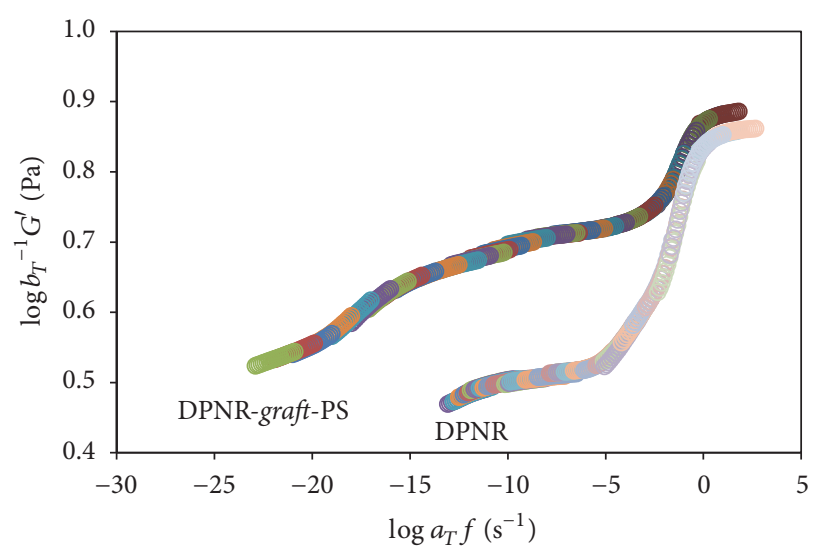

FIGURE 9: Master curves of storage modulus for DPNR and DPNRgraft-PS.

rubber indicates the pure entropic elasticity. However, in filled rubbers or thermoplastic elastomers, the interactions of polymer-polymer, polymer-filler, and filler-filler increase the intra- and intermolecular energy; then the energetic component contributes to the elasticity.

The previous work found that a positive slope in $b_{T}-T$ curve corresponds to entropic elasticity, in case of natural rubber $[27,38,40]$. On the contrary, a negative slope in $b_{T}-T$ curve corresponds to energetic elasticity, for instance, natural rubber filled by carbon black particles or natural rubber grafted vinyltriethoxysilane [35, 38-40]. Figure 10 shows the dependence of $b_{T}$ on the temperature of the DPNR and DPNR-graft-PS. $b_{T}$ of DPNR gradually increased along the temperature corresponding to the purely entropic behavior of natural rubber. Meanwhile, the curve of DPNR-graft-PS sample had the same positive slope of $b_{T}$ line, but the value of $b_{T}$ was lower than that of DPNR sample in temperature range from $T_{r}$ to $100^{\circ} \mathrm{C}$. These data can be rationalized in terms of graft copolymer having both entropic and energetic components. Above $100^{\circ} \mathrm{C}$, there was a prompt decrease in $b_{T}$. This can be explained to be due to the influence of the polystyrene component since DPNR-graft-PS has the higher glass transition temperature than the homopolystyrene. 


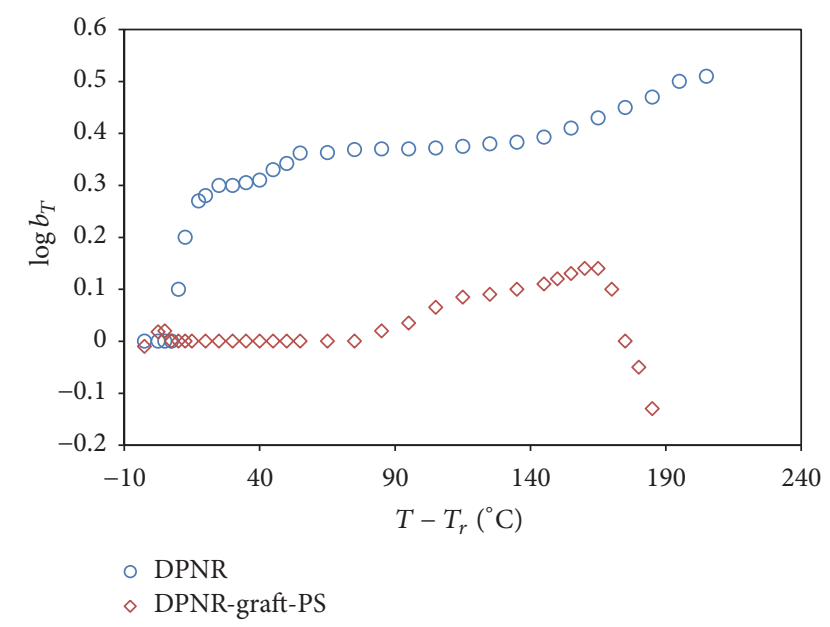

FIgURE 10: Temperature dependence of $b_{T}$ of DPNR and DPNRgraft-PS.

\section{Conclusions}

The dynamic mechanical properties of graft copolymer of natural rubber with styrene with nanomatrix structure were investigated at wide range of temperature by using timetemperature superposition principle. It was found that the storage modulus and loss modulus of the graft copolymers are dependent both on grafted polystyrene and on homopolystyrene content. The moduli of graft copolymer were found to increase with increasing total styrene content. The moduli of graft copolymer were increased with the increase of styrene contents. The working time of the graft copolymer was found to be longer and higher than that of the deproteinized natural rubber. It was proved by the master curves. It is also discovered that the graft copolymer has both energetic and entropic elasticity similar to the other common fillers, suggesting that graft copolymer could play a role as an organic filler for natural rubber when being applied by sinusoidal stress.

\section{Conflicts of Interest}

The authors declare that they have no conflicts of interest.

\section{Acknowledgments}

This research is funded by Vietnam National Foundation for Science and Technology Development (NAFOSTED) under Grant no. 104.04-2013.41.

\section{References}

[1] S. Zhang, J. Sun, X. Zhang, J. Xin, Q. Miao, and J. Wang, "Ionic liquid-based green processes for energy production," Chemical Society Reviews, vol. 43, no. 22, pp. 7838-7869, 2014.

[2] S. De, B. Saha, and R. Luque, "Hydrodeoxygenation processes: Advances on catalytic transformations of biomass-derived platform chemicals into hydrocarbon fuels," Bioresource Technology, vol. 178, pp. 108-118, 2015.
[3] L. Hu, L. Lin, Z. Wu, S. Zhou, and S. Liu, "Chemocatalytic hydrolysis of cellulose into glucose over solid acid catalysts," Applied Catalysis B: Environmental, vol. 174-175, pp. 225-243, 2015.

[4] J. I. Kroswit, Concise Encyclopedia of Polymer, Science Engineering, Wiley, London, England, 1990.

[5] H. F. Mark, Encyclopedia of Polymer Science Engineering, Wiley, New York, NY, USA, 1970.

[6] P. Wongthong, C. Nakason, Q. Pan, G. L. Rempel, and S. Kiatkamjornwong, "Modification of deproteinized natural rubber via grafting polymerization with maleic anhydride," European Polymer Journal, vol. 49, no. 12, pp. 4035-4046, 2013.

[7] S. Benmesli and F. Riahi, "Dynamic mechanical and thermal properties of a chemically modified polypropylene/natural rubber thermoplastic elastomer blend," Polymer Testing, vol. 36, pp. 54-61, 2014.

[8] S. Kovuttikulrangsie, K. Sahakaro, C. Intarakong, and P. Klinpituksa, "PMMA blended and DPNR-g-PMMA coated DPNR and NR-LA for dipping applications," Journal of Applied Polymer Science, vol. 93, no. 2, pp. 833-844, 2004.

[9] J. Madhanagopal, O. P. Singh, S. Sornambikai et al., "Enhanced wide-range monotonic piezoresistivity, reliability of Ketjenblack/deproteinized natural rubber nanocomposite, and its biomedical application," Journal of Applied Polymer Science, vol. 134, no. 25, Article ID 44981, 2017.

[10] J.-P. Zhong, S.-D. Li, Y.-C. Wei, Z. Peng, and H.-P. Yu, "Study on preparation of chlorinated natural rubber from latex and its thermal stability," Journal of Applied Polymer Science, vol. 73, no. 14, pp. 2863-2867, 1999.

[11] I. R. Gelling, "Modification of natural rubber latex with peracetic," Rubber Chemistry and Technology, vol. 58, no. 1, pp. 8696, 1985.

[12] A. Mahittikul, P. Prasassarakich, and G. L. Rempel, "'Hydrogenation of natural rubber latex in the presence of $\mathrm{OsHCl}(\mathrm{CO})\left(\mathrm{O}_{2}\right)\left(\mathrm{PCy}_{3}\right)_{2}$," Journal of Applied Polymer Science, vol. 100, no. 1, pp. 640-655, 2006.

[13] N. Huang and D. C. Sundberg, "Fundamental studies of grafting reactions in free radical copolymerization. I. A detailed kinetic model for solution polymerization," Journal of Polymer Science Part A: Polymer Chemistry, vol. 33, no. 15, pp. 2533-2549, 1995.

[14] N. Huang and D. C. Sundberg, "Fundamental studies of grafting reactions in free radical copolymerization. IV. Grafting of styrene, acrylate, and methacrylate monomers onto vinyl-polybutadiene using benzoyl peroxide and AIBN initiators in solution polymerization.," Journal of Polymer Science Part A: Polymer Chemistry, vol. 33, no. 15, pp. 2587-2603, 1995.

[15] F. E. Okieimen and I. N. Urhoghide, "Graft copolymerization of acrylonitrile and methyl methacrylate monomer mixtures on crumb natural rubber," Journal of Applied Polymer Science, vol. 84, no. 10, pp. 1872-1877, 2002.

[16] P. Prasassarakich, W. Arayapranee, and G. L. Rempel, "Synthesis of graft copolymers from natural rubber using cumene hydroperoxide redox initiator," Journal of Applied Polymer Science, vol. 83, no. 14, pp. 2993-3001, 2002.

[17] K. Songsing, T. Vatanatham, and N. Hansupalak, "Kinetics and mechanism of grafting styrene onto natural rubber in emulsion polymerization using cumene hydroperoxidetetraethylenepentamine as redox initiator," European Polymer Journal, vol. 49, no. 5, pp. 1007-1016, 2013.

[18] S. Kawahara, T. Kawazura, T. Sawada, and Y. Isono, "Preparation and characterization of natural rubber dispersed in nanomatrix," Polymer Journal, vol. 44, no. 16, pp. 4527-4531, 2003. 
[19] L. Fukuhara, N. Kado, N. T. Thuong et al., "Nanomatrix structure formed by graft copolymerization of styrene onto fresh natural rubber," Rubber Chemistry and Technology, vol. 88, no. 1, pp. 117-124, 2015.

[20] K. P. Menard, Dynamic mechanical analysis: A practical introduction, CRC press, Florida, USA, 1st edition, 1999.

[21] J. D. Ferry, Viscoelastic Properties of Polymers, John Wiley \& Sons, Canada, 3rd edition, 1980.

[22] E. N. Lawrence and F. L. Robert, Mechanical Properties of Polymers and Composites, Marcel Dekker, Inc, New York, NY, USA, 2nd edition, 1994.

[23] M. Doi and S. F. Edwards, The Theory of Polymer Dynamicsm, Clarendon Press, Oxford, UK, 1986.

[24] T. A. Dung, N. T. Nhan, N. T. Thuong et al., "Modification of Vietnam natural rubber via graft copolymerization with styrene," Journal of the Brazilian Chemical Society, vol. 28, no. 4, pp. 669-675, 2017.

[25] M. L. Williams, R. F. Landel, and J. D. Ferry, "The temperature dependence of relaxation mechanisms in amorphous polymers and other glass-forming liquids," Journal of the American Chemical Society, vol. 77, no. 14, pp. 3701-3707, 1955.

[26] J. E. Mark, Physical Properties of Polymers Handbook, Springer, New York, NY, USA, 2nd edition, 2007.

[27] M. Takahashi, M. C. Shen, R. B. Taylor, and A. V. Tobolsky, "Master curves for some amorphous polymers," Journal of Applied Polymer Science, vol. 8, no. 4, pp. 1549-1561, 1964.

[28] S. Kawahara, W. Klinklai, H. Kuroda, and Y. Isono, "Removal of proteins from natural rubber with urea," Polymers for Advanced Technologies, vol. 15, no. 4, pp. 181-184, 2004.

[29] W. Klinklai, T. Saito, S. Kawahara et al., "Hyperdeproteinized natural rubber prepared with urea," Journal of Applied Polymer Science, vol. 93, no. 2, pp. 555-559, 2004.

[30] N. Pukkate, Y. Yamamoto, and S. Kawahara, "Mechanism of graft copolymerization of styrene onto deproteinized natural rubber," Colloid and Polymer Science, vol. 286, no. 4, pp. 411-416, 2008.

[31] M. Klüppel, "Evaluation of viscoelastic master curves of filled elastomers and applications to fracture mechanics," Journal of Physics: Condensed Matter, vol. 21, no. 3, Article ID 035104, 2009.

[32] N. Jouault, P. Vallat, F. Dalmas, S. Said, J. Jestin, and F. Boue, "Well-dispersed fractal aggregates as filler in polymersilica nanocomposites: Long-range effects in rheology," Macromolecules, vol. 42, no. 6, pp. 2031-2040, 2009.

[33] D. Derouet, Q. N. Tran, and J. L. Leblanc, "Physical and mechanical properties of foly(methyl methacrylate)-grafted natural rubber synthesized by methyl methacrylate photopolymerization initiated by N,N-diethyldithiocarbamate functions previously created on natural rubber chains," Journal of Applied Polymer Science, vol. 112, no. 2, pp. 788-799, 2009.

[34] L. Dagdug and L. S. García-Colín, "Generalization of the Williams-Landel-Ferry equation," Physica A: Statistical Mechanics and its Applications, vol. 250, no. 1-4, pp. 133-141, 1998.

[35] T. Tada, K. Urayama, T. Mabuchi, K. Muraoka, and T. Takigawa, "Nonlinear stress relaxation of carbon black-filled rubber vulcanizates under various types of deformation," Journal of Polymer Science Part B: Polymer Physics, vol. 48, no. 12, pp. 13801387, 2010.

[36] Y. Isono and T. Aoyama, "Filler effects on temperature shift factors in viscoelastic properties of carbon black filled rubbers," Nihon Reoroji Gakkaishi, vol. 41, no. 3, pp. 137-144, 2013.
[37] A. P. Holt, J. R. Sangoro, Y. Wang, A. L. Agapov, and A. P. Sokolov, "Chain and segmental dynamics of poly(2-vinylpyridine) nanocomposites," Macromolecules, vol. 46, no. 10, pp. 4168-4173, 2013.

[38] J. Wu, S. Fujii, S. Kawahara, and Y. Isono, "Filler Effects on Temperature Dependence of Viscoelastic Properties of Filled Rubbers," e-Journal of Soft Materials, vol. 3, pp. 41-48, 2007.

[39] S. Kawahara, N. H. Yusof, K. Noguchi, K. Kosugi, and Y. Yamamoto, "Organic-inorganic nanomatrix structure and properties of related naturally occurring rubbery macromolecules," Polymer (United Kingdom), vol. 55, no. 20, pp. 5024-5027, 2014.

[40] N. H. Yusof, K. Noguchi, L. Fukuhara, Y. Yamamoto, and S. Kawahara, "Preparation and properties of natural rubber with filler nanomatrix structure," Colloid and Polymer Science, vol. 293, no. 8, pp. 2249-2256, 2015. 

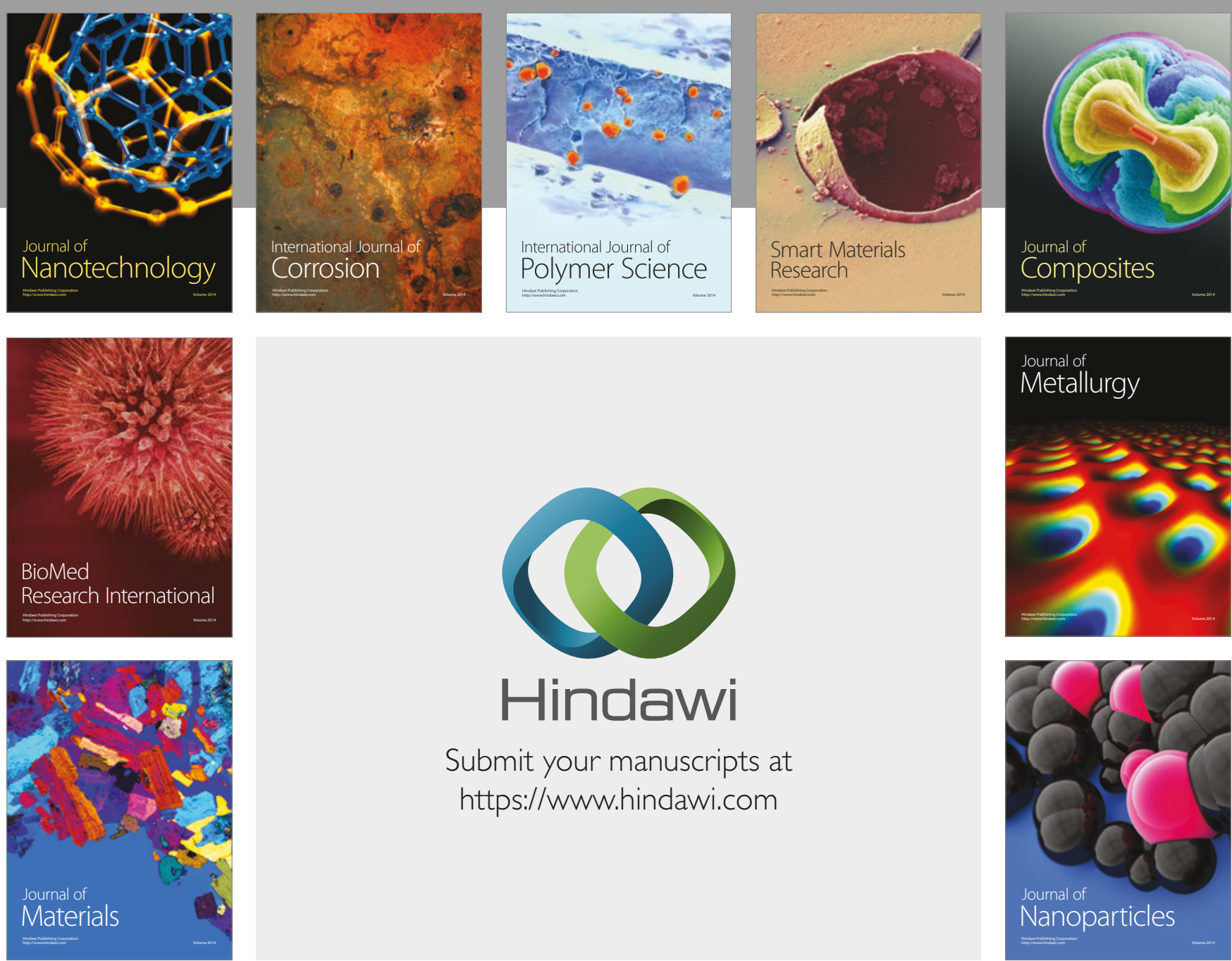

\section{Hindawi}

Submit your manuscripts at

https://www.hindawi.com
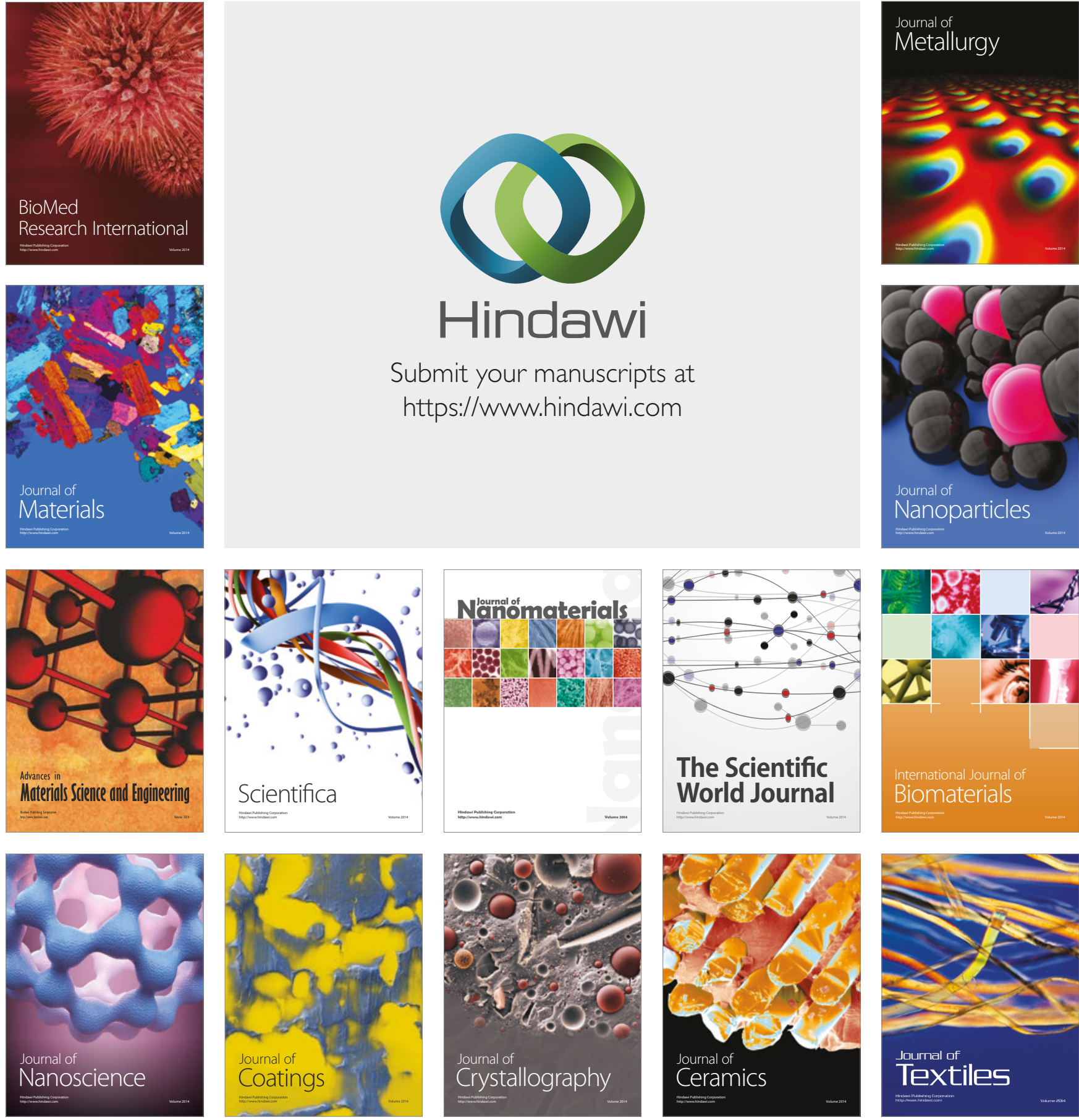

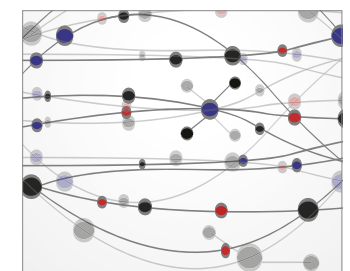

The Scientific World Journal
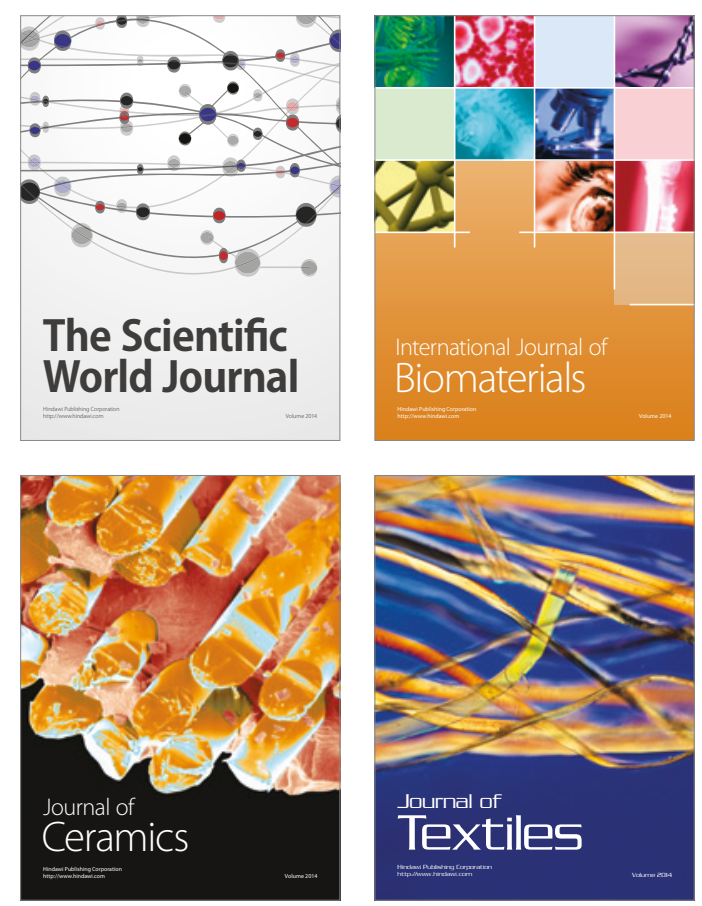